\title{
The Significance of Soil Micromorphology Research in Land Reclamation
}

\author{
Tingting Cao ${ }^{1,2,3,4, *}$, Jian Wang ${ }^{1,2,3,4}$, Haiou Zhang ${ }^{1,2,3,4}$ \\ ${ }^{1}$ Shaanxi Land Engineering Construction Group Co., Ltd. Xi'an 710075, China; \\ ${ }^{2}$ Institute of Land Engineering and Technology, Shaaxi Land Engineering Construction Group Co., Ltd. Xi'an 710075, China; \\ ${ }^{3}$ Key Laboratory of Degraded and Unused Land Consolidation Engineering, the Ministry of Natural Resources, Xi'an 710075, China \\ ${ }^{4}$ Shaanxi Land Consolidation Engineering Technology Research Center, Xi'an 710075, China
}

\begin{abstract}
Soil micromorphology is an emerging branch of soil science that aims to study the formation and structure of soils at a microscopic level. The study of soil micromorphology can be used for the analysis of soil genesis and evolution, the development of land remediation techniques, the analysis of the causes of geological hazards, the mechanisms of soil quality degradation, the process of soil formation and material changes in "anthropogenic soils" and many other aspects. With the gradual development of instrumentation, scanning electron microscopy, light microscopy and scanning CT have been widely used in the study of soil micromorphology. This paper presents a detailed analysis of the current state of development of soil micromorphology techniques and the possible problems that may exist in the future.
\end{abstract}

Key words: Soil micromorphology; Micro structure; Land reclamation

\section{1. introduction}

With economic development, the intensity and breadth of exploitation of land resources is becoming more and more obvious, and the conflict between man and land is becoming more and more prominent. Remediation of difficult-to-use land and degraded land to make it meet the real needs of resource exploitation, increase the area of arable land, improve food production and ensure food output is a scientific problem that needs to be solved urgently in land engineering nowadays. Shaanxi Province, for example, is high in the north and south and low in the centre, with the Shaanxi-North Plateau in the north, the Guanzhong Plain in the centre and the Qinba Mountains in the south. According to the classification of landforms, Shaanxi Province can be divided into six landform type regions: the Wind and Sand Transition Region, the Loess Plateau Region, the Guanzhong Plain Region, the Qinling Mountain Region, the Han River Basin Region and the Daba Mountain Region. In different landscape environments, soil properties are inevitably different and the problems that need to be solved differ, but the objective of remediation of degraded and difficult-to-use land is the same, i.e. to turn these difficult-to-use and unused lands into usable, high-standard and high-yielding agricultural land through scientific land engineering remediation methods.

Land degradation is a major scientific problem that limits agricultural production, affects ecological security, and threatens the natural environment with the development and utilization of land resources at a certain stage of social development. In response to this scientific problem, domestic and foreign scholars and associations have carried out relevant research, paid sufficient attention to the obvious degradation of soil, and carried out fruitful scientific research on various obvious degradation problems, and achieved remarkable results. However, up to now, enough attention has not been paid to the recessive degradation of soil, and there is a lack of knowledge about its occurrence mechanism and its harmfulness, and the occurrence mechanism has not yet been proved.

In Shaanxi Province, for example, the soil quality in the Guanzhong region of Shaanxi is excellent, and most of the unused land in this region needs to be remediated using techniques such as land restoration, raw soil ripening and concentrated patchwork. In southern Shaanxi, the remediation of unused land requires techniques such as soil and water conservation, viscosity reduction and soil loosening. The northern Shaanxi windsand region is a key area of concern because it has a vast land area and is one of the most promising areas for the development of arable land resources in Shaanxi Province, but due to the impact of local ecological problems such as wind and sand erosion and soil erosion, the land is infertile and food production is not high. The sandy land leaks water and fertiliser severely, making it difficult for vegetation to attach and grow. However, the arsenic sandstone, which is widely distributed in Yulin, is as hard as stone without water and as soft as mud with water, and is easily eroded by water and wind. Both are important sources of local

\footnotetext{
*Corresponding author: 956874403@qq.com
} 
ecological problems. One study has made full use of the characteristics of their soil properties to mix them in optimal proportions, i.e. to compensate for the poor soil structure of sandy soils and to make full use of the local arsenic sandstone colloidal material. The result is a soil with a good structure for crop growth, which can be improved in other ways using organic soil reconstruction techniques, resulting in a new land remediation technology that can be used for large-scale conversion.

The development of this land remediation technology is based on the full use of soil micromorphology research, i.e. the development of a new land remediation engineering technology, and also reveals the scientific mechanism of this land remediation technology from a microscopic point of view, making up the basic theory of the new land remediation technology and laying a solid scientific foundation for the positive development of the effects of land remediation. The research results are important for enhancing the social benefits of land remediation technology. The ecological and economic benefits also have important strategic significance.

\section{The current state of development of soilmicromorphology techniques}

Soil micromorphology is an emerging branch of soil science that aims to study soil formations and formation structures at the microscopic level, mainly by using high magnification instruments and equipment to observe the original microscopic morphology of the soil, and at present, the widely used equipment mainly includes scanning electron microscopy, optical microscopy, scanning CT and other instruments. The main objective is to observe the morphology of soil skeletal particles, finegrained material, soil basal structures and soil formations, as well as the configuration, structure and spatial distribution of the components of each type of particle, and to study the occurrence and formation mechanisms of soil micromorphology. This research tool is now widely used in land remediation techniques, analysis of the causes of geological disasters, mechanisms of soil quality degradation, soil formation processes and material changes in "anthropogenic soils".

In the analysis of soil micromorphological elements, the skeletal particles of soil micromorphology are classified as skeletal particles if they are larger than 2 microns. It is difficult to change the composition of the soil within a short period of time.

Soil fine-grained material consists mainly of soil mucilage components and humus. The fine-grained material of the soil consists almost exclusively of clay minerals, which aggregate to form the soil matrix. The structure of the soil matrix refers to the relevant distribution of each of the skeletal particles in the soil, as well as the size distribution of the skeletal particles and the state of existence of the fine-grained material in terms of shape and size as a form of organisation. There are various types of soil formations, including soil propertyrelated formations, depletion formations, crystalline formations, amorphous and cryptocrystalline formations, basal formations and excretion formations. The first four formation types are related to the soil parent rock and its developmental processes and are not significantly influenced by roots under short-term plant growth.

This shows that soil microstructure plays a key role in determining soil quality, with different soil structures directly affecting the amount of water stored in the soil, the amount of gas held and the nutrient storage. Different soil microstructure characteristics also have a direct impact on the penetration capacity of the root system, which in turn affects the contact surface of the plant with the subsoil and the efficiency of the use of nutrients in the soil. The study of soil microstructure can therefore contribute to a deeper understanding of soil formation processes and their changes, and provide an important basis for the development of land use, management and improvement measures. There are many methods for studying soil microstructure, which can be broadly divided into direct and indirect methods depending on the nature of the indicators used to characterise the microform of the soil, and depending on the equipment used and the method of study, the information used to characterise soil microstructure varies. One of the most common devices used to observe soil micromorphology is the scanning electron microscope, which, at different magnifications, can be used to obtain soil micromorphology at different scales and provide a more intuitive view of the morphological information of the soil structure.

For a good soil configuration, the cementing material is the key to soil formation and the key material for the formation of soil agglomerates. This agglomeration force refers to the bonding of soil particles to each other through cohesion. The formation of this force can lead to an increase in the forces between the soil particles, so that the soil has sufficient resistance to external forces, of which the longest interparticle forces are mainly Van der Waals forces, Coulomb forces and surface tension. Because of the key role of colloidal forces in the soil formation process, the components of colloids, in their distributed form, are crucial for the development of soil succession. Soils are developed and stabilised by a combination of subtle forces within the soil. The research results on soil colloidal substances and microstructure are gradually being enriched in China. With the continuous improvement of research methods, the state of the soil basement is studied on a microscopic scale with the aid of scanning electron microscopy, light and electron microscopy, the mode of action of colloidal substances is analysed, and the composition of organic carbon functional groups is analysed semi-quantitatively by means of synchrotron radiation. In addition, soil structure has been studied using computed tomography, nuclear magnetic resonance and corresponding image processing techniques to investigate soil agglomerates, the relationship between cementing substances and soil structure and function, etc. However, due to the complexity of soil types and soil-forming environments, there is no universal theory of agglomerate formation processes and mechanisms.

Raman spectroscopy is a type of scattering spectroscopy and has been used in recent years in the field of soil science to study land engineering techniques. When a sample is examined, the sample is irradiated by a laser and 
the scattered laser light is generated by the interaction between the rotation of the sample molecules and the laser, which mainly includes Rayleigh scattering, Torx scattering and anti-Stokes scattering. Raman spectroscopy instruments can detect mainly the antiStokes scattering. Different substances have their own unique Raman spectral lines. The information on the position of the characteristic peaks, the full width of the half-peaks and the intensity of the peaks in the spectrum can be used for the analysis of the structure, state and special properties of the soil. Raman spectroscopy has a wide range of applications and plays an important role in the fields of materials, biology, environmental protection and geology. The analysis of soil samples using Raman spectroscopy enables a more in-depth and detailed study of soil structure and properties at the microscopic level.

\section{Conclusion}

With the increasing importance of basic research, the study of soil micromorphology from a microscopic perspective plays a vital role in improving the basic theory of soil science and aiding scientific and technological research and development in the field of land remediation techniques. Research in this area is becoming an important branch of the discipline. The study of soil microstructure provides a visual representation of soil conditions on a microscopic scale, and the results can be used to analyse soil genesis and evolution, as well as for land remediation techniques, analysis of the causes of geological hazards, mechanisms of soil quality degradation, soil formation processes and material changes in "man-made soils", depending on the purpose of the research. Research. Due to the instrumentation and sample preparation requirements for the observation of soil microstructure, only a small number of samples are required to observe the microform of the soil, so the representativeness of the sampling needs to be further explored, and a comprehensive description of the overall soil profile based on the results of a spot scan of a small number of samples requires a large number of observations to be compared to obtain a scientific and objective result. As the micromorphological properties of soils require the observer to compare the results of the images based on what has been observed, combined with specialist knowledge, to obtain specific results on soil micromorphology, the observer's control of specialist knowledge, and image recognition techniques are also essential for qualitative conclusions on soil micromorphology.

\section{Acknowledgments}

This study is financially supported by the Natural Science Basic Research Program of Shaanxi (2021JZ-57);

Shaanxi Province Innovative Talents Program-Youth Science and Technology Rising Star Project (2021KJXX88)

\section{References}

1. Cao Mingjie, Hao Zhe, Yang Jingshuai, Xiang Tingting, Ni Junkang, Xu Ying, Kaibi. Effects of different improvement measures on soil micromorphology in drainage sites[J]. Nonferrous Metal Engineering,2020,10(05):96-102.

2. Zhang Baohua,Tao Baoxian,Cao Jianrong,Liu Ziting. Pore micromorphological characteristics of tidal soils in the lower Yellow River alluvial plain[J]. Geography of Arid Regions,2020,43(03):687-693.

3. Yang Jianjun,Wang Yihao,Wang Jian, $\mathrm{Hu}$ Yongfeng.Progress and prospects of microscale heavy metal soil chemistry research $[\mathrm{J}]$. Journal of Soil Science,2020,57(03):530-539.

4. Charles S, Wu KN, Liang SY, Zhuang DC. Advances in the study of paleoanthropogenic activities based on site soil analysis[J]. Soil Bulletin,2019,50(04):958964.

5. Zhang Baohua,Tao Baoxian,Liu Ziting,CaoJianrong. Micromorphological characteristics of different textured tidal soils in the lower Yellow River alluvial plain[J]. Shandong Agricultural Science,2019,51(07):67-71.

6. Li Zhenzhen,Wang Shu,Zhang Hongfei,Mao shuailei,REN Naou. Micromorphological study on the effect of clay on sandy soil improvement in the horqin sandy area of Inner Mongolia[J]. Journal of China Agricultural University,2018,23(11):115-123.

7. Ye Xiqiong. Study on the removal characteristics of petroleum hydrocarbons by microbial remediation and environmental changes of soil microbiology[D]. Xi'an University of Architecture and Technology,2018.

8. Hu Hui. Comparative study on the micromorphology of loess-paleosols in the north and south of the Qinling Mountains[D]. Shaanxi Normal University,2018.

9. $\mathrm{Hu}$ Hui,Pang Jiangli,Huang Chunchang,Zha Xiaochun,Zhou Yali,Mao Peini. Comparison of micromorphological characteristics of loesspaleosols in the north and south of the Qinling Mountains[J]. Soil Bulletin,2018,49(02):275-282.

10. Liu Ying, Wang Shu, Zhang Fengrong, Lv Yizhong, Li Zhenzhen, Mao shuailei,REN Naou. Micromorphological evaluation of fertility of sandy soils under different improvement measures: the case of horqin sandy land in Inner Mongolia[J]. Soil,2017,49(05):1023-1031.

11. Bai Yang, Chen Yiqian, Zhang Xiaohui. Study on the microstructure and mechanical properties of paleosols from landslides in freeze-thaw cycles [J]. People's Yangtze River, 2021, 52(07): 192-197+203. DOI: 10.16232/j.cnki .1001-4179.2021.07.032.

12. Luo Xilian, Wang Yike, Han Jichang, Gu Zhaolin. The effect of experimental period on the microstructure of arsenic sandstone-improved aeolian soil [J]. Journal of Xi'an Jiaotong University, 2021, 55(12): 172-179. 
13. Wu Jiangpeng. Study on the influence of temperature and salt solution on the microstructure of clay based on nuclear magnetic resonance [D]. Guilin University of Technology, 2021.

14. Zhao Tianxiang. Study on the effect of grass ash on improving the mechanical stability of sandy subgrade slopes [D]. Inner Mongolia Agricultural University, 2021. DOI: 10.27229/d.cnki.gnmnu.2021.000429.

15. Huang Jianjia. Effects of free iron oxide on shear and consolidation strength of granite residual soil [D]. Huazhong Agricultural University, 2021.

16. Wang Huaixing. Experimental study on microbial solidification and erosion resistance of loess [D]. University of Chinese Academy of Sciences (Research Center for Soil and Water Conservation and Ecological Environment, Ministry of Education, Chinese Academy of Sciences), 2021.DOI:10.27558/d.cnki.gsthc.2021.000036.

17. Dong Shaowen, Ma Shuhua, Chu Mo, Wang Xiaohui, Wang Yuejiao, Liu Chenxu, Han Fenglan. Changes in the microstructure of saline-alkali soil under the action of fly ash-based soil conditioners [J]. Chinese Journal of Process Engineering: 1-9[2022- 01-12].

18. Guo Lina, Zhang Yongbo, Duan Qing, Shi Hong, Wu Aijing. A review of the research on the mechanical properties of soil modified by nanomaterials [J]. Water-Saving Irrigation, 2021(03):79-84.

19. Bai Linjie, Yin Song, Wang Jin, Li Xinming, Li Li. Experimental study on microstructural properties of silt under freeze-thaw cycles [J]. Journal of Zhongyuan University of Technology, 2021,32(01):55-59+73. 